Network Working Group

Request for Comments: 5657

$\mathrm{BCP}: 9$

Updates: 2026

Category: Best Current Practice
L. Dusseault

Messaging Architects

R. Sparks

Tekelec

September 2009

\title{
Guidance on Interoperation and Implementation Reports for Advancement to Draft Standard
}

\section{Abstract}

Advancing a protocol to Draft standard requires documentation of the interoperation and implementation of the protocol. Historic reports have varied widely in form and level of content and there is little guidance available to new report preparers. This document updates the existing processes and provides more detail on what is appropriate in an interoperability and implementation report.

Status of This Memo

This document specifies an Internet Best Current Practices for the Internet Community, and requests discussion and suggestions for improvements. Distribution of this memo is unlimited.

Copyright and License Notice

Copyright (c) 2009 IETF Trust and the persons identified as the document authors. All rights reserved.

This document is subject to BCP 78 and the IETF Trust's Legal Provisions Relating to IETF Documents (http://trustee.ietf.org/license-info) in effect on the date of publication of this document. Please review these documents carefully, as they describe your rights and restrictions with respect to this document. Code Components extracted from this document must include Simplified BSD License text as described in section $4 . e$ of the Trust Legal Provisions and are provided without warranty as described in the BSD License. 
Table of Contents

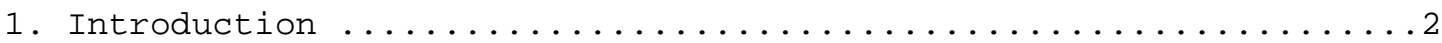

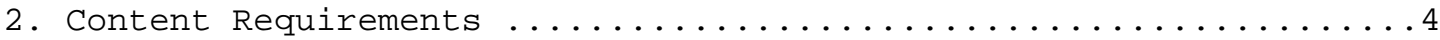

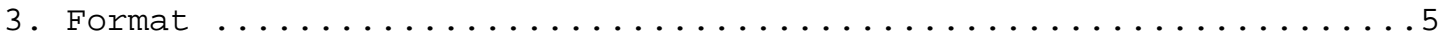

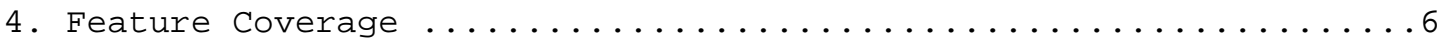

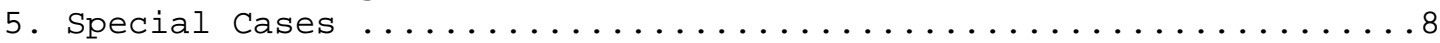

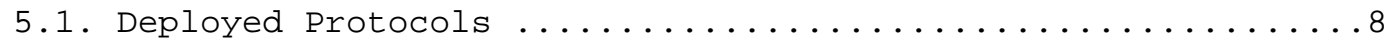

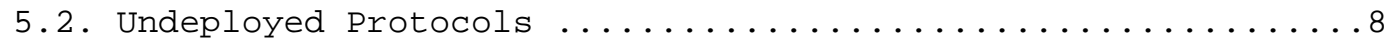

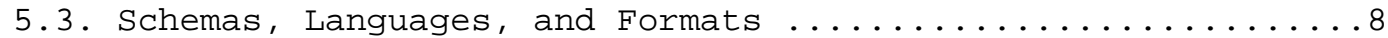

5.4. Multiple Contributors, Multiple Implementation Reports ....9

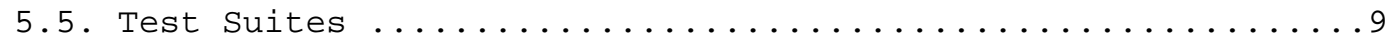

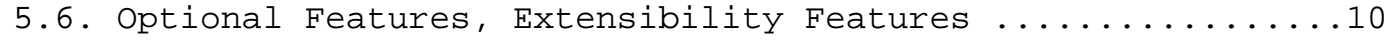

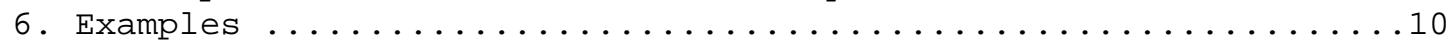

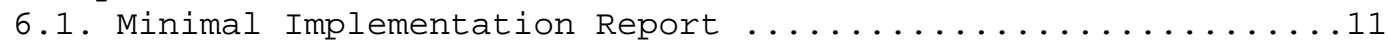

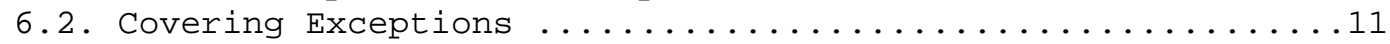

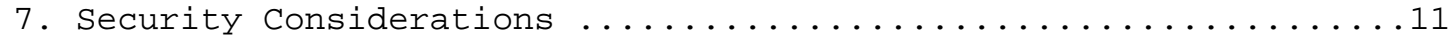

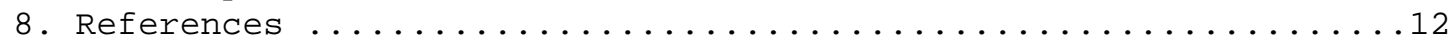

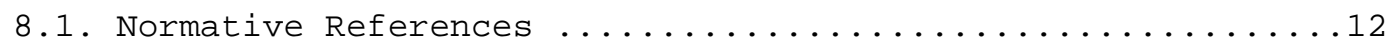

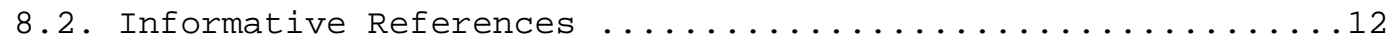

\section{Introduction}

The Draft Standard level, and requirements for standards to meet it, are described in [RFC2026]. For Draft Standard, not only must two implementations interoperate, but also documentation (the report) must be provided to the IETF. The entire paragraph covering this documentation reads:

The Working Group chair is responsible for documenting the specific implementations which qualify the specification for Draft or Internet standard status along with documentation about testing of the interoperation of these implementations. The documentation must include information about the support of each of the individual options and features. This documentation should be submitted to the Area Director with the protocol action request. (see Section 6)

Moving documents along the standards track can be an important signal to the user and implementor communities, and the process of submitting a standard for advancement can help improve that standard or the quality of implementations that participate. However, the barriers seem to be high for advancement to Draft standard, or at the very least confusing. This memo may help in guiding people through one part of advancing specifications to Draft standard. It also changes some of the requirements made in RFC 2026 in ways that are intended to maintain or improve the quality of reports while reducing the burden of creating them. 
Having and demonstrating sufficient interoperability is a gating requirement for advancing a protocol to Draft standard. Thus, the primary goal of an implementation report is to convince the IETF and the IESG that the protocol is ready for Draft standard. This goal can be met by summarizing the interoperability characteristics and by providing just enough detail to support that conclusion. Side benefits may accrue to the community creating the report in the form of bugs found or fixed in tested implementations, documentation that can help future implementors, or ideas for other documents or future revisions of the protocol being tested.

Different kinds of documentation are appropriate for widely deployed standards than for standards that are not yet deployed. Different test approaches are appropriate for standards that are not typical protocols: languages, formats, schemas, etc. This memo discusses how reports for these standards may vary in section 5.

Implementation should naturally focus on the final version of the RFC. If there's any evidence that implementations are interoperating based on Internet-Drafts or earlier versions of the specification, or if interoperability was greatly aided by mailing list clarifications, this should be noted in the report.

The level of detail in reports accepted in the past has varied widely. An example of a submitted report that is not sufficient for demonstrating interoperability is (in its entirety): "A partial list of implementations include: Cray SGI Netstar IBM HP Network Systems Convex". This report does not state how it is known that these implementations interoperate (was it through public lab testing? internal lab testing? deployment?). Nor does it capture whether implementors are aware of, or were asked about, any features that proved to be problematic. At a different extreme, reports have been submitted that contain a great amount of detail about the test methodology, but relatively little information about what worked and what failed to work.

This memo is intended to clarify what an implementation report should contain and to suggest a reasonable form for most implementation reports. It is not intended to rule out good ideas. For example, this memo can't take into account all process variations such as documents going to Draft standard twice, nor can it consider all types of standards. Whenever the situation varies significantly from what's described here, the IESG uses judgement in determining whether an implementation report meets the goals above.

The key words "MUST", "MUST NOT", "REQUIRED", "SHALL", "SHALL NOT", "SHOULD", "SHOULD NOT", "RECOMMENDED", "MAY", and "OPTIONAL" in this document are to be interpreted as described in BCP 14 [RFC2119]. 


\section{Content Requirements}

The implementation report MUST identify the author of the report, who is responsible for characterizing the interoperability quality of the protocol. The report MAY identify other contributors (testers, those who answered surveys, or those who contributed information) to share credit or blame. The report MAY provide a list of report reviewers who corroborate the characterization of interoperability quality, or name an active working group (WG) that reviewed the report.

Some of the requirements of RFC 2026 are relaxed with this update:

- The report MAY name exactly which implementations were tested. A requirement to name implementations was implied by the description of the responsibility for "documenting the specific

implementations" in RFC 2026. However, note that usually identifying implementations will help meet the goals of implementation reports. If a subset of implementations was tested or surveyed, it would also help to explain how that subset was chosen or self-selected. See also the note on implementation independence below.

- The report author MAY choose an appropriate level of detail to document feature interoperability, rather than document each individual feature. See note on granularity of features below.

- A contributor other than a WG chair MAY submit an implementation report to an Area Director (AD).

- Optional features that are not implemented, but are important and do not harm interoperability, MAY, exceptionally and with approval of the IESG, be left in a protocol at Draft standard. See Section 5.6 for documentation requirements and an example of where this is needed.

Note: Independence of implementations is mentioned in the RFC 2026 requirements for Draft Standard status. Independent implementations should be written by different people at different organizations using different code and protocol libraries. If it's necessary to relax this definition, it can be relaxed as long as there is evidence to show that success is due more to the quality of the protocol than to out-of-band understandings or common code. If there are only two implementations of an undeployed protocol, the report SHOULD identify the implementations and their "genealogy" (which libraries were used or where the codebase came from). If there are many more implementations, or the protocol is in broad deployment, it is not necessary to call out which two of the 
implementations demonstrated interoperability of each given feature -- a reader may conclude that at least some of the implementations of that feature are independent.

Note: The granularity of features described in a specification is necessarily very detailed. In contrast, the granularity of an implementation report need not be as detailed. A report need not list every "MAY", "SHOULD", and "MUST" in a complete matrix across implementations. A more effective approach might be to characterize the interoperability quality and testing approach, then call out any known problems in either testing or interoperability.

3. Format

The format of implementation and interoperability reports MUST be ASCII text with line breaks for readability. As with InternetDrafts, no 8-bit characters are currently allowed. It is acceptable, but not necessary, for a report to be formatted as an Internet-Draft.

Here is a simple outline that an implementation report MAY follow in part or in full:

Title: Titles of implementation reports are strongly RECOMMENDED to contain one or more RFC number for consistent lookup in a simple archive. In addition, the name or a common mnemonic of the standard should be in the title. An example might look like "Implementation Report for the Example Name of Some Protocol (ENSP) RFC XXXX".

Author: Identify the author of the report.

Summary: Attest that the standard meets the requirements for Draft Standard and name who is attesting it. Describe how many implementations were tested or surveyed. Quickly characterize the deployment level and where the standard can be found in deployment. Call out, and if possible, briefly describe any notably difficult or poorly interoperable features and explain why these still meet the requirement. Assert any derivative conclusions: if a high-level system is tested and shown to work, then we may conclude that the normative requirements of that system (all sub-system or lower-layer protocols, to the extent that a range of features is used) have also been shown to work.

Methodology: Describe how the information in the report was obtained. This should be no longer than the summary. 
Exceptions: This section might read "Every feature was implemented, tested, and widely interoperable without exception and without question". If that statement is not true, then this section should cover whether any features were thought to be problematic. Problematic features need not disqualify a protocol from Draft Standard, but this section should explain why they do not (e.g., optional, untestable, trace, or extension features). See the example in Section 6.2 .

Detail sections: Any other justifying or background information can be included here. In particular, any information that would have made the summary or methodology sections more than a few paragraphs long may be created as a detail section and referenced.

In this section, it would be good to discuss how the various considerations sections played out. Were the security considerations accurate and dealt with appropriately in implementations? Was real internationalization experience found among the tested implementations? Did the implementations have any common monitoring or management functionality (although note that documenting the interoperability of a management standard might be separate from documenting the interoperability of the protocol itself)? Did the IANA registries or registrations, if any, work as intended?

Appendix sections: It's not necessary to archive test material such as test suites, test documents, questionnaire text, or questionnaire responses. However, if it's easy to preserve this information, appendix sections allow readers to skip over it if they are not interested. Preserving detailed test information can help people doing similar or follow-on implementation reports, and can also help new implementors.

\section{Feature Coverage}

What constitutes a "feature" for the purposes of an interoperability report has been frequently debated. Good judgement is required in finding a level of detail that adequately demonstrates coverage of the requirements. Statements made at too high a level will result in a document that can't be verified and hasn't adequately challenged that the testing accidentally missed an important failure to interoperate. On the other hand, statements at too fine a level result in an exponentially exploding matrix of requirement interaction that overburdens the testers and report writers. The important information in the resulting report would likely be hard to find in the sea of detail, making it difficult to evaluate whether the important points of interoperability have been addressed. 
The best interoperability reports will organize statements of interoperability at a level of detail just sufficient to convince the reader that testing has covered the full set of requirements and in particular that the testing was sufficient to uncover any places where interoperability does not exist. Reports similar to that for RTP/RTCP (an excerpt appears below) are more useful than an exhaustive checklist of every normative statement in the specification.

10. Interoperable exchange of receiver report packets.

- PASS: Many implementations, tested UCL rat with vat, Cisco IP/TV with vat/vic.

11. Interoperable exchange of receiver report packets when not receiving data (ie: the empty receiver report which has to be sent first in each compound RTCP packet when no-participants are transmitting data).

o PASS: Many implementations, tested UCL rat with vat, Cisco IP/TV with vat/vic.

・・

8. Interoperable transport of RTP via TCP using the encapsulation defined in the audio/video profile

- FAIL: no known implementations. This has been removed from the audio/video profile.

\footnotetext{
Excerpts from

http://www.ietf.org/iesg/implementation/report-avt-rtp-rtcp.txt

Consensus can be a good tool to help determine the appropriate level for such feature descriptions. A working group can make a strong statement by documenting its consensus that a report sufficiently covers a specification and that interoperability has been demonstrated.
} 


\section{Special Cases}

\subsection{Deployed Protocols}

When a protocol is deployed, results obtained from laboratory testing are not as useful to the IETF as learning what is actually working in deployment. To this end, it may be more informative to survey implementors or operators. A questionnaire or interview can elicit information from a wider number of sources. As long as it is known that independent implementations can work in deployment, it is more useful to discover what problems exist, rather than gather long and detailed checklists of features and options.

\subsection{Undeployed Protocols}

It is appropriate to provide finer-grained detail in reports for protocols that do not yet have a wealth of experience gained through deployment. In particular, some complicated, flexible or powerful features might show interoperability problems when testers start to probe outside the core use cases. RFC 2026 requires "sufficient successful operational experience" before progressing a standard to Draft, and notes that:

Draft Standard may still require additional or more widespread field experience, since it is possible for implementations based on Draft Standard specifications to demonstrate unforeseen behavior when subjected to large-scale use in production environments.

When possible, reports for protocols without much deployment experience should anticipate common operational considerations. For example, it would be appropriate to put additional emphasis on overload or congestion management features the protocol may have.

\subsection{Schemas, Languages, and Formats}

Standards that are not on-the-wire protocols may be special cases for implementation reports. The IESG SHOULD use judgement in what kind of implementation information is acceptable for these kinds of standards. ABNF (RFC 4234) is an example of a language for which an implementation report was filed: it is interoperable in that protocols are specified using $A B N F$ and these protocols can be successfully implemented and syntax verified. Implementations of ABNF include the RFCs that use it as well as ABNF checking software. Management Information Base (MIB, [RFC3410]) modules are sometimes documented in implementation reports, and examples of that can be found in the archive of implementation reports. 
The interoperability reporting requirements for some classes of documents may be discussed in separate documents. See [METRICSTEST] for example.

5.4. Multiple Contributors, Multiple Implementation Reports

If it's easiest to divide up the work of implementation reports by implementation, the result -- multiple implementation reports -- MAY be submitted to the sponsoring Area Director one-by-one. Each report might cover one implementation, including:

identification of the implementation;

an affirmation that the implementation works in testing (or better, in deployment);

whether any features are known to interoperate poorly with other implementations;

which optional or required features are not implemented (note that there are no protocol police to punish this disclosure, we should instead thank implementors who point out unimplemented or unimplementable features especially if they can explain why); and

who is submitting this report for this implementation.

These SHOULD be collated into one document for archiving under one title, but can be concatenated trivially even if the result has several summary sections or introductions.

\subsection{Test Suites}

Some automated tests, such as automated test clients, do not test interoperability directly. When specialized test implementations are necessary, tests can at least be constructed from real-world protocol or document examples. For example:

- ABNF [RFC4234] itself was tested by combining real-world examples -- uses of ABNF found in well-known RFCs -- and feeding those real-world examples into ABNF checkers. As the well-known RFCs were themselves interoperable and in broad deployment, this served as both a deployment proof and an interoperability proof. [RFC4234] progressed from Proposed Standard through Draft Standard to standard and is obsoleted by [RFC5234]. 
- Atom [RFC4287] clients might be tested by finding that they consistently display the information in a test Atom feed, constructed from real-world examples that cover all the required and optional features.

- MIB modules can be tested with generic MIB browsers, to confirm that different implementations return the same values for objects under similar conditions.

As a counter-example, the automated WWW Distributed Authoring and Versioning (WebDAV) test client Litmus

(http://www.webdav.org/neon/litmus/) is of limited use in demonstrating interoperability for WebDAV because it tests completeness of server implementations and simple test cases. It does not test real-world use or whether any real WebDAV clients implement a feature properly or at all.

\subsection{Optional Features, Extensibility Features}

Optional features need not be shown to be implemented everywhere. However, they do need to be implemented somewhere, and more than one independent implementation is required. If an optional feature does not meet this requirement, the implementation report must say so and explain why the feature must be kept anyway versus being evidence of a poor-quality standard.

Extensibility points and versioning features are particularly likely to need this kind of treatment. When a protocol version 1 is released, the protocol version field itself is likely to be unused. Before any other versions exist, it can't really be demonstrated that this particular field or option is implemented.

6. Examples

Some good, extremely brief, examples of implementation reports can be found in the archives:

http://wWw.ietf.org/iesg/implementation/report-ppp-lcp-ext.html

http://www.ietf.org/iesg/implementation/report-otp.html

In some cases, perfectly good implementation reports are longer than necessary, but may preserve helpful information:

http://wWw.ietf.org/iesg/implementation/report-rfc2329.txt

http://www.ietf.org/iesg/implementation/report-rfc4234.txt 
6.1. Minimal Implementation Report

A large number of SMTP implementations support SMTP pipelining, including: (1) Innosoft's PMDF and Sun's SIMS. (2) ISODE/ MessagingDirect's PP. (3) ISOCOR's nPlex. (4) software.com's post.office. (5) Zmailer. (6) Smail. (7) The SMTP server in Windows 2000. SMTP pipelining has been widely deployed in these and other implementations for some time, and there have been no reported interoperability problems.

This implementation report can also be found at http://www. ietf.org//iesg/implementation/report-smtp-pipelining.txt but the entire report is already reproduced above. Since SMTP pipelining had no interoperability problems, the implementation report was able to provide all the key information in a very terse format. The reader can infer from the different vendors and platforms that the codebases must, by and in large, be independent.

This implementation report would only be slightly improved by a positive affirmation that there have been probes or investigations asking about interoperability problems rather than merely a lack of problem reports, and by stating who provided this summary report.

\subsection{Covering Exceptions}

The RFC2821bis (SMTP) implementation survey asked implementors what features were not implemented. The VRFY and EXPN commands showed up frequently in the responses as not implemented or disabled. That implementation report might have followed the advice in this document, had it already existed, by justifying the interoperability of those features up front or in an "exceptions" section if the outline defined in this memo were used:

VRFY and EXPN commands are often not implemented or are disabled. This does not pose an interoperability problem for SMTP because EXPN is an optional features and its support is never relied on. VRFY is required, but in practice it is not relied on because servers can legitimately reply with a non-response. These commands should remain in the standard because they are sometimes used by administrators within a domain under controlled circumstances (e.g. authenticated query from within the domain). Thus, the occasional utility argues for keeping these features, while the lack of problems for end-users means that the interoperability of SMTP in real use is not in the least degraded.

7. Security Considerations

This memo introduces no new security considerations. 
8. References

8.1. Normative References

[RFC2119] Bradner, S., "Key words for use in RFCs to Indicate Requirement Levels", BCP 14, RFC 2119, March 1997.

8.2. Informative References

[METRICSTEST] Bradner, S. and V. Paxson, "Advancement of metrics specifications on the IETF Standards Track", Work in Progress, July 2007.

[RFC2026] Bradner, S., "The Internet Standards Process -Revision 3", BCP 9, RFC 2026, October 1996.

[RFC3410] Case, J., Mundy, R., Partain, D., and B. Stewart, "Introduction and Applicability Statements for Internet-Standard Management Framework", RFC 3410, December 2002 .

[RFC4234] Crocker, D., Ed. and P. Overell, "Augmented BNF for Syntax Specifications: ABNF", RFC 4234, October 2005.

[RFC4287] Nottingham, M., Ed. and R. Sayre, Ed., "The Atom Syndication Format", RFC 4287, December 2005.

[RFC5234] Crocker, D. and P. Overell, "Augmented BNF for Syntax Specifications: ABNF", STD 68, RFC 5234, January 2008.

Authors' Addresses

Lisa Dusseault

Messaging Architects

EMail: lisa.dusseault@gmail.com

Robert Sparks

Tekelec

17210 Campbell Road

Suite 250

Dallas, Texas 75254-4203

USA

EMail: RjS@nostrum.com 